\title{
Systemic administration of 6-OHDA to rhesus monkeys upregulates HLA-DR expression in brain microvasculature
}

This article was published in the following Dove Press journal:

Journal of Inflammation Research

18 September 2014

Number of times this article has been viewed

\author{
Valerie Joers ${ }^{1,2}$ \\ Scott Vermilyea ${ }^{1,2}$ \\ Kristine Dilley' \\ Marina E Emborg ${ }^{1-3}$ \\ 'Preclinical Parkinson's Research \\ Program, Wisconsin National \\ Primate Research Center, \\ ${ }^{2}$ Neuroscience Training Program, \\ ${ }^{3}$ Department of Medical Physics, \\ University of Wisconsin-Madison, \\ Madison, WI, USA
}

Correspondence: Marina E Emborg

Preclinical Parkinson's Research Program,

Wisconsin National Primate Research

Center, University of Wisconsin,

Madison, 1223 Capitol Court,

Madison, WI 53715, USA

$\mathrm{Tel}+\mathrm{I} 6082629714$

Fax + I 6082633524

Email emborg@primate.wisc.edu
Background: We recently developed a nonhuman primate model of cardiac dysautonomia by systemic dosing of the catecholaminergic neurotoxin 6-hydroxydopamine (6-OHDA). The aim of this study was to assess whether systemic 6-OHDA affects the central nervous system of nonhuman primates, in particular the dopaminergic nigrostriatal system.

Methods: Brain sections from adult rhesus monkeys that received systemic 6-OHDA $(50 \mathrm{mg} / \mathrm{kg}$ intravenously; $n=5)$ and were necropsied 3 months later, as well as normal controls $(n=5)$ were used in this study. Tissue was cut frozen at $40 \mu \mathrm{m}$ on a sliding microtome, processed for immunohistochemistry, and blindly evaluated.

Results: Neither the optical density of tyrosine hydroxylase immunoreactivity (TH-ir; a dopaminergic neuronal marker) in the caudate and putamen nucleus nor the TH-ir cell number and volume in the substantia nigra showed significant differences between groups. Yet within groups, statistical analysis revealed significant individual differences in the 6-OHDA-treated group, with two animals showing a lower cell count and volume. Optical density quantification of $\alpha$-synuclein-ir in the substantia nigra did not show differences between groups. As $\alpha$-synuclein intracellular distribution was noted to vary between animals, it was further evaluated with a semiquantitative scale. A greater intensity and presence of $\alpha$-synuclein-positive nigral cell bodies was associated with larger TH-positive nigral cell volumes. Increased human leukocyte antigen (HLA-DR; a microglial marker) expression was observed in 6-OHDA-treated animals compared with controls. HLA-DR-ir was primarily localized in endothelial cells and perivascular spaces throughout cortical and subcortical structures. Semiquantitative evaluation using a rating scale revealed higher HLA-DR-ir in blood vessels of 6-OHDA-treated animals than controls, specifically in animals with the lowest number of dopaminergic nigral neurons.

Conclusion: Our results demonstrate that systemic 6-OHDA administration to rhesus monkeys can affect the dopaminergic nigrostriatal system and upregulate inflammatory markers in the cerebrovasculature that persist 3 months post neurotoxin challenge. The variability of the subject response suggests differences in individual sensitivity to 6-OHDA.

Keywords: 6-hydroxydopamine, blood-brain barrier, nonhuman primates, neuroinflammation, parkinsonism

\section{Introduction}

Parkinson's disease is a progressive neurodegenerative multisystem disorder that presents both motor and nonmotor symptoms. The pathological hallmarks of Parkinson's disease are the loss of dopaminergic nigral neurons and the presence of intracytoplasmic inclusions termed Lewy bodies, which are mainly composed by the presynaptic protein $\alpha$-synuclein. Neuroinflammation, observed as activated microglia and reactive astrocytes, is also typically found in the brains of patients with Parkinson's disease. ${ }^{1-4}$ 
6-Hydroxydopamine (6-OHDA) is a catecholaminergic neurotoxin used to model Parkinson's disease in animals. Similar to dopamine, 6-OHDA does not cross the blood-brain barrier $^{5,6}$ and its method of administration defines whether central or peripheral catecholaminergic neurons are affected, and therefore which symptoms of Parkinson's disease are modeled. When 6-OHDA is intracerebrally injected into the nigrostriatal system or medial forebrain bundle, it replicates typical loss of dopaminergic nigral neurons in Parkinson's disease. ${ }^{7-12}$ Systemic dosing of 6-OHDA induces loss of catecholaminergic innervation to the heart and adrenal medulla, ${ }^{13}$ and replicates Parkinson's disease pathologies associated with cardiac dysautonomia. There are no reports of 6-OHDA-induced $\alpha$-synuclein changes in monkey models, yet in 1-methyl-4-phenyl-1,2,3,6-tetrahydropyridine (MPTP)treated monkeys, investigators have found aggregation and redistribution of $\alpha$-synuclein, suggesting that neurotoxins have the potential to interact with $\alpha$-synuclein. ${ }^{14,15}$

Although systemic dosing of 6-OHDA to rodents does not seem to affect central catecholamines, it has been shown to compromise the blood-brain barrier in areas of the mesencephalon. ${ }^{16}$ We have recently developed a nonhuman primate model of cardiac dysautonomia by systemic dosing of 6-OHDA. ${ }^{17,18}$ In vivo and post mortem analysis confirmed loss of catecholaminergic innervation in the heart as well as loss of circulating catecholamines produced by the adrenal medulla; motor symptoms of Parkinson's disease were not observed. Here we report the brain post mortem studies to assess whether systemic administration of 6-OHDA to rhesus monkeys has central nervous system effects, in particular in the dopaminergic nigrostriatal network.

\section{Material and methods Ethics statement}

The present study was performed in strict accordance with the recommendations in the National Institutes of Health Guide for the Care and Use of Laboratory Animals (1996) in an Association for Assessment and Accreditation of Laboratory Animal Care accredited facility (Wisconsin National Primate Research Center, University of Wisconsin-Madison). Experimental procedures were approved by the Institutional Animal Care and Use Committee of the University at the Wisconsin-Madison (permit number G00538). All efforts were made to minimize the number of animals used and to ameliorate any distress.

\section{Subjects}

Brain sections from nine adult rhesus monkeys (Macaca mulatta, aged 5-17 years) were utilized for this study.
The animals from which the tissue was obtained were individually housed in group 3 or group 4 enclosures in accordance with the Animal Welfare Act and the Guide for the Care and Use of Laboratory Animals (7th edition, 1996) with a 12-hour light/dark cycle. Throughout the study, the animals were monitored twice daily by an animal research or veterinary technician for evidence of disease or injury (eg, lack of appetite, dehydration, diarrhea, lethargy, trauma), and body weight was monitored to ensure animals remained in properly sized cages. Animals were fed commercial nonhuman primate chow (2050 Teklad global 20\% protein primate diet; Harlan Laboratories, Madison, WI, USA) twice daily, supplemented with fruit or vegetables and a variety of forage items. and received water ad libitum. Nonhuman primate chow soaked in a protein-enriched drink (Ensure ${ }^{\circledR}$; Abbott Laboratories, Abbott Park, IL, USA) was offered to stimulate appetite as needed.

Normal brain sections were obtained from four adult rhesus monkeys (mean age 9.6 \pm 5.2 years, mean weight $11.03 \pm 3.29 \mathrm{~kg}$ ) from the Wisconsin National Primate Center tissue bank. The 6-OHDA-treated brain sections were from five rhesus monkeys (mean age $7.0 \pm 1.57$ years, $7.35 \pm 1.65 \mathrm{~kg}$ ) from a previously published study. ${ }^{18}$ Dosing of 6-OHDA to these monkeys was done in sterile surgical conditions under isoflurane anesthesia, as a sequence of 7-9 injections totaling a final dose of $50 \mathrm{mg} / \mathrm{kg}$. The animals were necropsied 3 months post 6 -OHDA.

\section{Necropsy and preparation of tissue}

Animals from which the tissue was obtained were anesthetized with sodium pentobarbital sodium ( $35 \mathrm{mg} / \mathrm{kg}$, intravenously) and perfused through the ascending aorta artery with heparinized phosphate-buffered saline, followed by $4 \%$ paraformaldehyde. The brains were removed from the calvaria, blocked and immersed in 4\% paraformaldehyde for 48-72 hours, and cryoprotected in increasing grades $(10 \%-30 \%)$ of sucrose $/ 0.1$ $\mathrm{M}$ phosphate-buffered saline ( $\mathrm{pH}$ 7.2) solution. Tissue slabs were frozen and cut at $40 \mu \mathrm{m}$ on a sliding microtome and the tissue stored in cryoprotectant as previously described. ${ }^{19}$

\section{Clinical rating scale evaluation}

All monkeys were monitored daily and any changes in overall health and general behaviors, including presence of neurological signs, were recorded. Animals that received 6-OHDA were evaluated for symptoms of parkinsonism using a previously validated clinical rating scale. ${ }^{20,21}$ Briefly, the scale assessed tremor, posture, gait, bradykinesia, balance, gross motor skills, defense reaction, and freezing 
using a 32-point scale, with a higher score describing a severe disability. Assessments were recorded at baseline and approximately 4, 10, and 14 weeks after 6-OHDA as previously reported. ${ }^{18}$

\section{General immunohistochemistry}

Immunohistochemical staining of brain sections was performed as described in previously published protocols ${ }^{19,22}$ for tyrosine hydroxylase (TH; 10,000; Immunostar, Hudson, WI, USA), microglia marker human B-lymphocyte LN-3 (HLA-DR; 1:200; MP Biomedical, Santa Ana, CA, USA), glial fibrillary acidic protein (GFAP; 1:2,000; DakoCytomation, Glostrup, Denmark), and $\alpha$-synuclein (Invitrogen, Carlsbad, CA, USA). HLA-DR staining was enhanced by addition of ammonium nickel sulfate. Tissue was immunostained for soluble and insoluble $\alpha$-synuclein using the protocol described above. Immunostaining for insoluble $\alpha$-synuclein included an additional incubation in proteinase K (1:4,000; Life Technologies, Grand Island, NY, USA) for 10 minutes before endogenous peroxidase block. All slides were dehydrated and coverslipped with permount (Fisher Scientific, Pittsburgh, PA, USA) and dried overnight.

\section{Immunofluorescence}

Sequential day immunofluorescence stainings were performed in brain sections to identify the localization of vascular (by CD31) and microglia (by HLA-DR) markers. Tissue was blocked in $5 \%$ donkey serum and $2 \%$ bovine serum albumin before primary antibody incubation with mouse anti-CD31 (1:80; DakoCytomation) and 0.1\% Triton X-100 overnight at $4^{\circ} \mathrm{C}$. Tissue was incubated in donkey anti-mouse immunoglobulin G conjugated to Alexa Fluor-488 (1:1000; Invitrogen). Tissue was washed and incubated in a second serum block containing $5 \%$ goat serum and $2 \%$ bovine serum albumin, following the second primary antibody mouse antiHLA-DR (1:100) with $0.1 \%$ Triton X-100 overnight at $4^{\circ} \mathrm{C}$. Tissue was incubated in Alexa Fluor-594-conjugated goat anti-mouse secondary (1:1,000; Invitrogen). All tissues were incubated in 4' 6-diamidino-2-phenylindole dihydrochloride (DAPI) for 5 minutes $(0.005 \mu \mathrm{g} / \mathrm{mL}$; Invitrogen $)$, mounted on subbed slides and coverslipped using fluorescent mounting medium (Golden Bridge International Inc., Mukiteo, WA, USA).

\section{Stereological TH-ir nigral cell counts}

The total number of neurons with TH immunoreactivity (-ir) in the right and left substantia nigra was determined using unbiased stereological cell counting methods as described previously. ${ }^{23,24}$ Briefly, the optical dissector system consisted of an AxioImager microscope (Zeiss, Oberkochen, Switzerland) coupled to a QImaging video camera system, and neuronal counts were performed using the optical fractionator probe in StereoInvestigator (MicroBrightField, Williston, VT, USA). Six equally spaced sections were used for analysis. The substantia nigra was outlined under a low magnification $(2.5 \times)$, and using dissector counting frames sized $150 \times 90 \mu \mathrm{m}$, tissue thickness was manually adjusted and neurons counted using a $63 \times$ oil immersion objective.

\section{Optical density quantification of TH-ir, soluble $\alpha$-synuclein-ir, and HLA-DR-ir}

Optical density (OD) was evaluated using National Institutes of Health ImageJ software version $1.43 \mathrm{u}$. TH-ir OD was quantified in six matched slides in the right and left caudate and putamen. HLA-DR-ir OD was evaluated in the right and left caudate and putamen in one matched slide and the substantia nigra in another matched slide. Soluble $\alpha$-synuclein OD was quantified in right and left substantia nigra in two slides. Images were captured using an Epson 1640XL-GA high-resolution digital scanner. ImageJ was calibrated using a step tablet, gray scale values converted to OD units using the Rodbard function, and the area above a threshold of 0.10 (TH) or 0.12 (HLA-DR and $\alpha$-synuclein) OD units was recorded.

\section{Evaluation of soluble and insoluble $\alpha$-synuclein-ir nigral distribution}

Soluble and insoluble $\alpha$-synuclein-ir in nigral cells were evaluated in two sections per staining using a light microscope (10×, Zeiss). Soluble $\alpha$-synuclein-ir in fibers and cell bodies was identified and scored separately using a semiquantitative scale. The scale was defined as $0=$ absent, $1=$ mild immunostaining, and $2=$ moderate immunostaining.

\section{Inflammation scale}

Two investigators blind to the treatment group evaluated tissue for HLA-DR-ir and GFAP-ir using a semiquantitative rating scale. Two coronal slices were assessed per animal per staining using a Zeiss light microscope under low magnification $(10 \times)$. One coronal slide at the level of the anterior commissure was used to evaluate the right and left putamen, caudate, internal capsule, septum, and anterior commissure. Another coronal slide at the level of cranial nerve III was used to assess the right and left thalamus, substantia nigra, and hippocampus. HLA-DR-positive microglia were defined as resting when cells presented small bodies 
and thin elongated processes (Figure 1A) and as activated when the cells had enlarged cell bodies and thickened or intensely immunostained processes (Figure 1B). Activated astrocytes were identified by GFAP-ir with intense hyperramified processes.

The rating scale for activated HLA-DR-ir and GFAP-ir was based on the number of focal patches of reactive microglia or astrocytes, respectively, including upregulated perivascular immunostaining (Figure $1 \mathrm{C}$ and $\mathrm{D}$ ). The scale was defined as $0=$ no staining, $1=$ weak (more than one focal patch), $2=$ moderate (more than two focal patches), $3=$ strong (more than three focal patches), and $4=$ intense (more than four focal patches).

HLA-DR-ir was further evaluated in resting microglia and blood vessels. The presence of HLA-DR-positive resting microglia and blood vessels expressing HLA-DR was evaluated using a semiquantitative scale defined as $0=$ absent, $1=$ weak, $2=$ moderate, and $3=$ strong.

\section{Statistical analysis}

Data collection and analysis were performed by investigators blind to the treatment groups. All statistical analysis was done using PASW Statistics version 18.0 software (SPSS Inc., Chicago, IL, USA) or Statistical Package for the Social Sciences version 18.0 software (SPSS Inc.). Morphological data were quantified and compared between control and 6-OHDA-treated monkeys using independent $t$-tests. Analysis within each group was conducted using one-way analysis of variance with Bonferroni post hoc

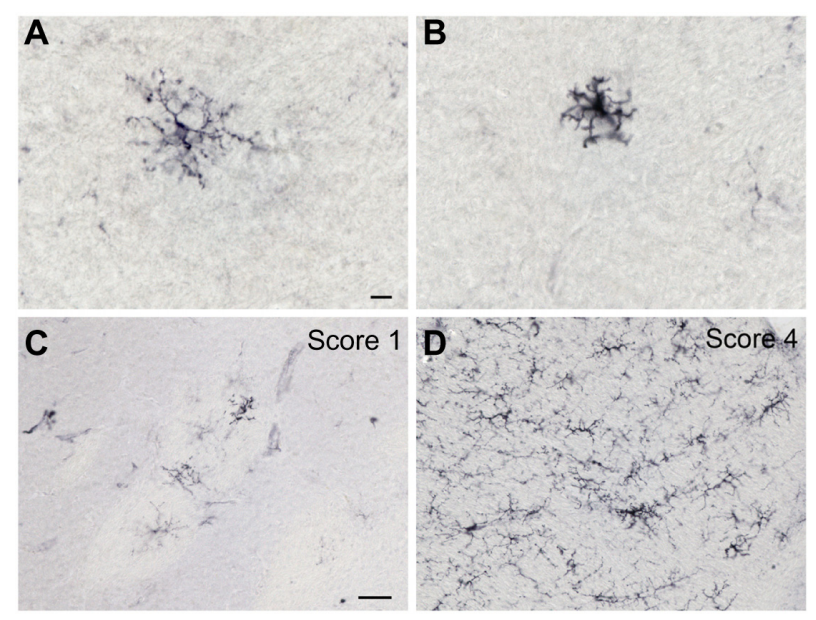

Figure I HLA-DR-ir microglia morphology and semiquantitative rating. Representative high magnification images of HLA-DR-positive resident (A) and activated (B) microglia. Characteristic images of HLA-DR-ir nigral sections showing a score of $I$ (C) and 4 (D) on a rating scale of $0-4$ defining the presence of activated microglia. Scale bar: $(\mathbf{A}$ and $\mathbf{B})=10 \mu \mathrm{m} ;(\mathbf{C}$ and $\mathbf{D})=50 \mu \mathrm{m}$.

Abbreviations: HLA, human leukocyte antigen; ir, immunoreactivity. analysis. Pearson's correlations were performed between morphological measures. Statistical significance was considered at $P<0.05$.

\section{Results}

\section{Parkinsonian motor signs were not detected after systemic 6-OHDA dosing}

All animals were highly active and interactive with their environment, displaying behaviors typical of adult rhesus monkeys. The 6-OHDA-treated animals did not display any signs of parkinsonism before or at any time point post neurotoxin, scoring 0 in the clinical rating scale.

\section{TH-ir was variably decreased in the substantia nigra of 6-OHDA-treated monkeys}

Qualitative evaluation of TH-ir revealed in all animals a similar pattern of intense expression of TH-positive fibers in the caudate and putamen (Figure 2A and B). Numerous TH-positive neurons were found within both the dorsal and ventral tier of the ventral mesencephalon and the ventral tegmental area, although some animals in the 6-OHDA group seemed to have decreased TH-ir (Figure $2 \mathrm{C}-\mathrm{E})$. TH-positive perikarya had round or triangular morphology, with multipolar varicose neurites emanating from the cell body. Dendrites from TH-ir somata were observed extending ventrally to the substantia nigra pars reticulata.

As comparisons per group between left and right OD TH-ir in the caudate and putamen or TH-positive nigral cell counts did not show significant differences, individual monkey datasets are reported as averages between left and right measures. OD quantification of TH-ir in the caudate and putamen showed no significant differences between groups. The average stereological TH-ir nigral cell counts were also not significantly different between groups (Figure 2F). Further analysis within the 6-OHDA group showed a significant difference in the number of TH-ir nigral cells $[\mathrm{F}(4,9)=18.68$, $P=0.0033$, with post hoc analysis demonstrating significance between r04094 and rh2318 $(P<0.05), \mathrm{r} 01098(P<0.05)$, and rh2316 ( $P<0.01$; Figure $2 \mathrm{G})$. Animals rh2316 and rh2318, in particular, had low nigral counts, suggesting differences in individual sensitivity to systemic 6-OHDA in the nigrostriatal system. Although animal r04094 had an average nigral cell count greater than the rest of the 6-OHDA group, a Grubb's outlier detection test did not find it an outlier (critical value, $\mathrm{Z}=1.715$; animal $\mathrm{r} 04094, \mathrm{Z}=1.5538 ; P>0.05$ ). No variability 
A
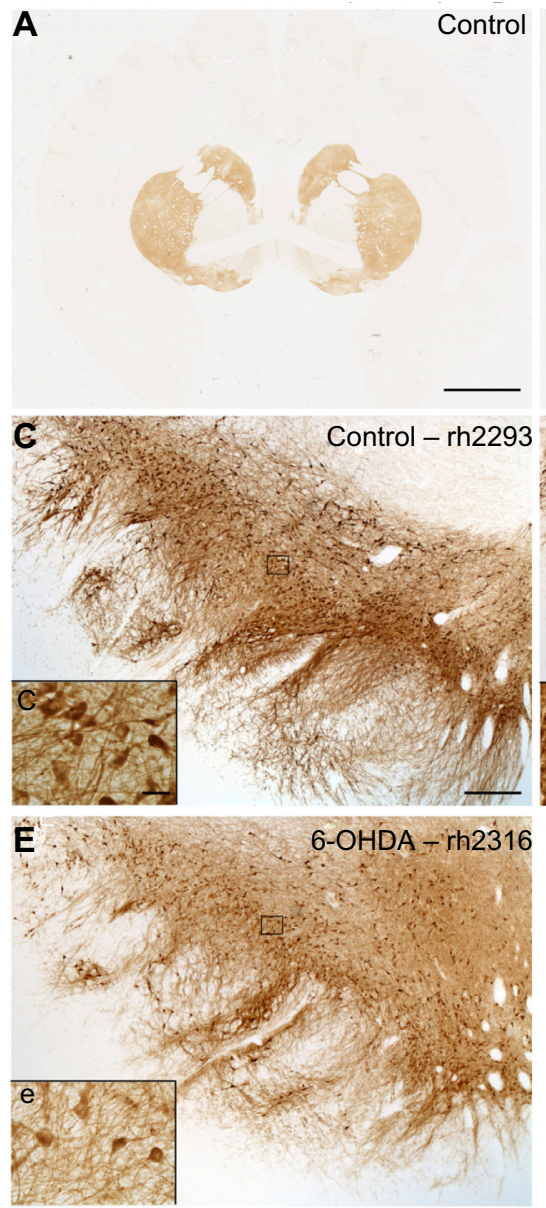

G

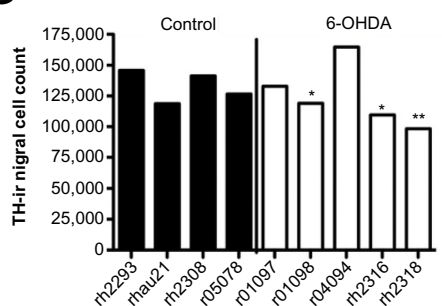

B

6-OHDA
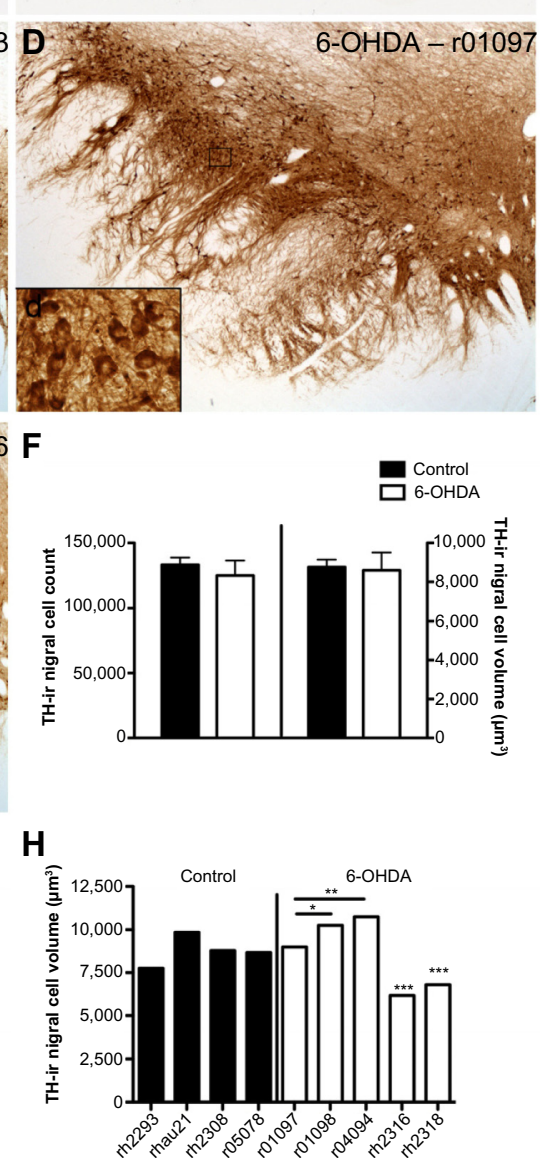

Figure 2 TH-ir nigral cell counts and volumes are variable in 6-OHDA-treated monkeys. Microphotographs of TH immunostained striatum at the level of the anterior commissure of control (A) and 6-OHDA-treated (B) monkeys, and in the left substantia nigra of a control (C, c), a 6-OHDA-treated monkey without reaction to toxin (D, d), and a 6-OHDA-treated monkey with a mild reaction to toxin (E, e). Stereological analysis of averaged TH-ir nigral cell counts and volumes show no difference between groups (F). TH-ir nigral cell counts for individual animals show no significant difference in the control group, but significant differences were found in 6-OHDAtreated animals, with $\mathrm{r01098,} \mathrm{rh2316,} \mathrm{and} \mathrm{rh2318} \mathrm{showing} \mathrm{a} \mathrm{smaller} \mathrm{number} \mathrm{of} \mathrm{nigral} \mathrm{cells} \mathrm{than} \mathrm{r04094} \mathrm{(G).} \mathrm{TH-ir} \mathrm{nigral} \mathrm{cell} \mathrm{volumes} \mathrm{for} \mathrm{individual} \mathrm{animals} \mathrm{show} \mathrm{no} \mathrm{significant}$ difference in the control group, but significant differences were found in 6-OHDA-treated animals, with a lower nigral volume found in r0I097 and r0I098 compared with r04094 and decreased volumes in rh2316 and rh2318 compared with all other 6-OHDA-treated animals $(\mathbf{H}) . * P<0.05, * * P<0.01, * * * P<0.001$. Scale bar: $(\mathbf{A}$ and $\mathbf{B})=10$ mm, $(\mathbf{C}-\mathbf{E})=500 \mu \mathrm{m}$; insets $=10 \mu \mathrm{m}$

Abbreviations: 6-OHDA, 6-hydroxydopamine; TH, tyrosine hydroxylase; ir, immunoreactivity.

in substantia nigra cell counts was found in the control group $[\mathrm{F}(3,7)=3.082, P=0.1527]$.

Differences in nigral cell volume were not detected in the right compared with the left substantia nigra in either the 6-OHDA group or the control group, suggesting no laterality in the size of cell bodies in the substantia nigra. The average volume of TH-ir nigral cells was not statistically different between groups (Figure 2F). Similar to TH-ir cell counts, significant variability was demonstrated within the 6-OHDA group for the nigral cell volume $[\mathrm{F}(4,9)=218.2, P<0.0001]$, with rh2316 and rh2318 having the smallest volumes of the 6-OHDA animals $(P<0.001$; Figure $2 \mathrm{H})$. The control group also had significant variability in TH-ir nigral cell volume $[\mathrm{F}(3,7)=7.071, P=0.0446]$, although a multiple comparisons test did not detect significance between animals. Averaged substantia nigra TH-ir cell counts did not correlate with 
the volumes of the same cells; however, trends were found when separating for the left $\left(r^{2}=0.436, P=0.053\right)$ and right $\left(r^{2}=0.359, P=0.088\right)$ substantia nigra.

\section{Intracellular distribution of $\alpha$-synuclein in nigral neurons varied within groups}

Soluble $\alpha$-synuclein-ir was present in the substantia nigra of all animals, localized in cell bodies and/or fibers extending ventrally. Individual differences were observed in the intensity of the staining and its distribution (Figure 3A and B). Control subjects had intense nigral $\alpha$-synuclein-ir in fibers, with the exception of one animal (aged 17 years and the eldest of the series) that showed only mild fiber expression, while all 6-OHDA-treated monkeys had mildly $\alpha$-synuclein-ir fibers. $\alpha$-Synuclein-ir in cell bodies was variably present in all monkeys, with the exception of one control monkey and one 6-OHDA-treated monkey that lacked any expression. None of the subjects had proteinase K-resistant, insoluble $\alpha$-synuclein aggregates.

Analysis of nigral soluble $\alpha$-synuclein-ir OD, in addition to scores distinguishing cellular and fiber soluble $\alpha$-synuclein-ir, did not show significant differences between treatment groups, probably due to the control subject that presented a pattern of staining similar to that in the 6-OHDA-treated animals.

Nigral $\alpha$-synuclein-ir OD (mean of right and left per animal) positively correlated with fiber scores $\left(r^{2}=0.893\right.$, $P<0.0001)$ and trended to negatively correlate with cell body scores $\left(r^{2}=0.368, P=0.083\right)$, suggesting that nigral $\alpha$-synuclein-ir OD is driven by expression of $\alpha$-synuclein in the fibers. Average soluble $\alpha$-synuclein cell body scores tended to positively correlate with average total volume of TH-ir in nigral cells $\left(r^{2}=0.437, P=0.053\right)$, suggesting that larger dopaminergic nigral cells had more soluble $\alpha$-synuclein.

\section{HLA-DR-ir was increased in the cerebrovasculature of 6-OHDA-treated monkeys}

GFAP-positive resident astrocytes were observed in all animals. A few reactive astrocytes exhibiting enlarged cell

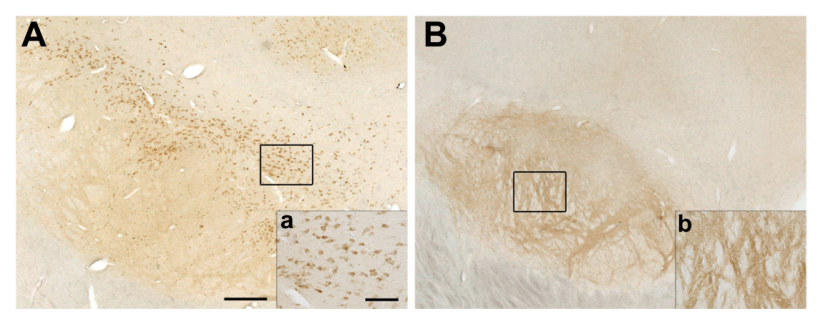

Figure 3 Distribution of $\alpha$-synuclein-ir varies in the substantia nigra. Representative images of $\alpha$-synuclein immunostaining in nigral cell bodies $(\mathbf{A}, \mathbf{a})$ or fibers $(\mathbf{B}, \mathbf{b})$. Scale bar: $(\mathbf{A}$ and $\mathbf{B})=500 \mu \mathrm{m} ;(\mathbf{a}$ and $\mathbf{b})=50 \mu \mathrm{m}$. bodies with more intense GFAP-ir ramified morphology were found sparingly dispersed in white matter tracts, and in patches or surrounding large vasculature throughout nuclei. Semiquantitative analysis of GFAP-labeled reactive astrocytes showed no significant difference between groups.

In all animals, HLA-DR-ir microglia presented either as resting, typically displaying long, lightly immunoreactive fibers, or as mildly activated, characterized by enlarged cell bodies with increased cytoplasmic immunoreactivity and hyper-ramified fibers; activated cells tended to cluster together. HLA-DR-ir cells were found in the anterior commissure, internal capsule, septum, and the substantia nigra, and to a lesser extent in the caudate, putamen, and hippocampus. Notably, 6-OHDA-treated monkeys also showed HLA-DR-positive cells with morphology resembling blood vessels throughout the striatal nuclei, substantia nigra, and hippocampus (Figure 4A-F). Localization of HLA-DR-ir in cerebral blood vessels of 6-OHDA-treated monkeys was confirmed with immunofluorescent colabeling of HLA-DR-ir and CD31-ir (Figure 5).

OD of HLA-DR-ir in the caudate, putamen, and substantia nigra, as well as scores for HLA-DR-ir in resting and activated microglia were not significantly different between groups. Interestingly, scoring for HLA-DR-ir in blood vessels demonstrated a significant increase in the caudate $[\mathrm{t}(7)=3.388, P=0.012]$, septum $[\mathrm{t}(7)=2.657, P=0.033]$, and hippocampus $[\mathrm{t}(4.739)=2.788, P=0.041]$ in 6-OHDA-treated animals compared with controls (Figure 4G) suggesting that systemic 6-OHDA dosing induced upregulation of HLADR-ir in the cerebrovasculature that persisted 3 months post administration. Other brain regions showed trends towards increased HLA-DR-ir scores in blood vessels of 6-OHDAtreated animals compared with control animals [putamen: $\mathrm{t}(7)=2.123, P=0.071$; thalamus: $\mathrm{t}(7)=2.106, P=0.073]$.

The amount of nigral HLA-DR-ir in blood vessels negatively correlated with the number of nigral TH-ir cells $\left(r^{2}=0.563, P=0.02\right)$ and trended toward a significant negative correlation with the volume of $\mathrm{TH}$-ir in the substantia nigra $\left(r^{2}=0.389, P=0.072\right)$.

\section{Discussion}

The present study demonstrates that systemic 6-OHDA dosing in rhesus monkeys variably affects the central dopaminergic nigrostriatal system and induces a proinflammatory response in the cerebrovasculature. To the best of our knowledge, this is the first report in nonhuman primates assessing central effects of systemic dosing of 6-OHDA.

6-OHDA is a hydroxylated analog of dopamine and toxic to catecholamine-producing cells. Similar to the 

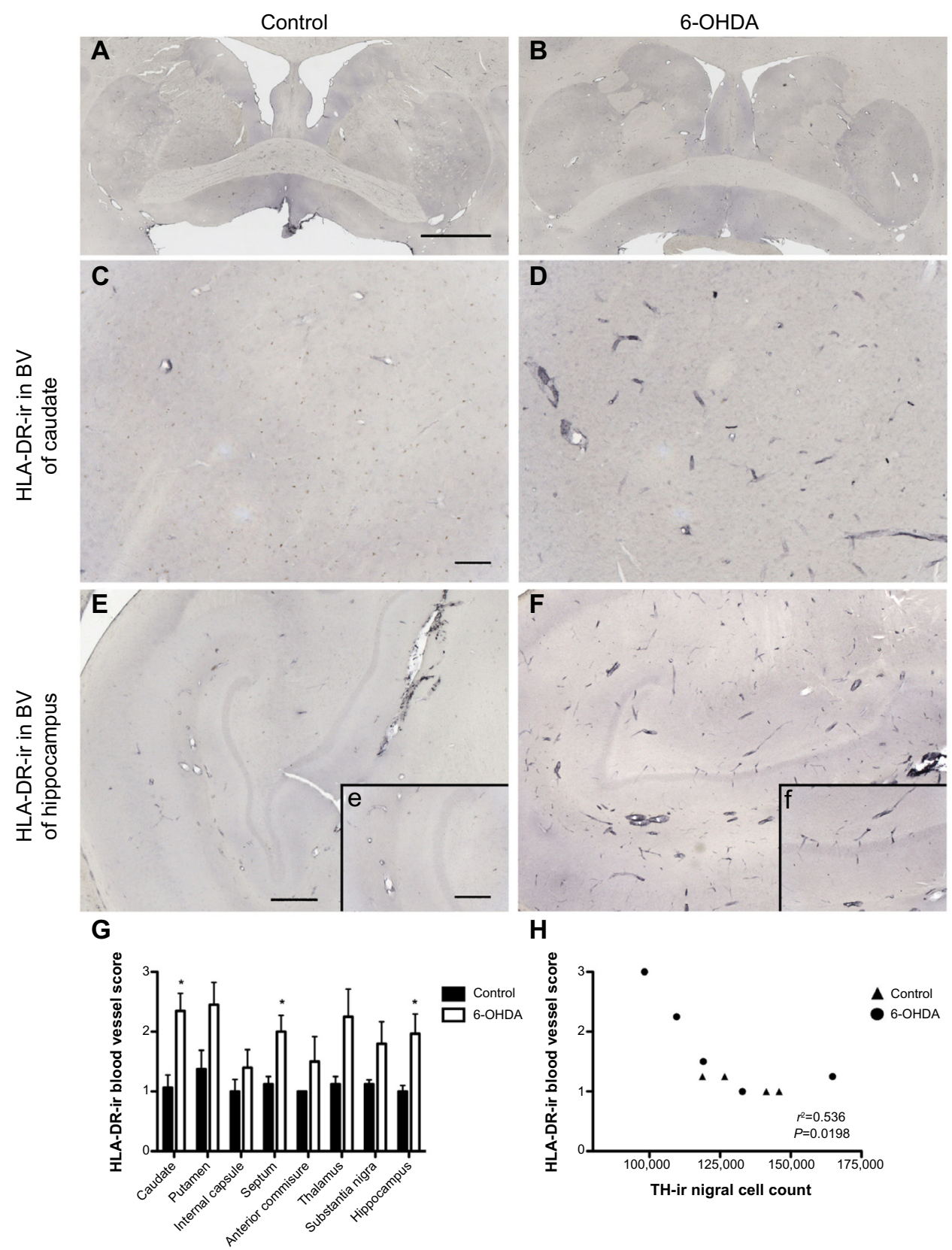

H

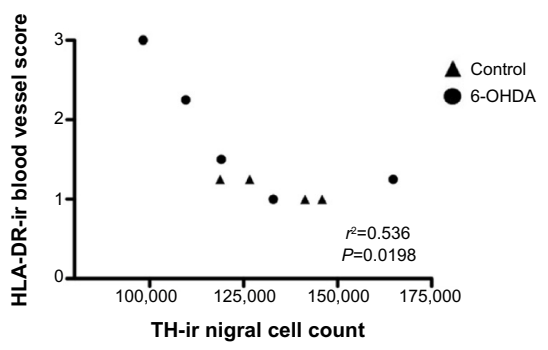

Figure 4 Increased expression of HLA-DR in blood vessels of 6-OHDA-treated monkeys. Microphotographs of HLA-DR immunostained striatum at the level of the anterior commissure of a control monkey $(\mathbf{A})$ and a 6-OHDA-treated monkey (B). Mild HLA-DR expression was found in the cerebrovasculature of a control animal in the caudate $(\mathbf{C})$ and hippocampus (E, e), while HLA-DR-ir was upregulated in 6-OHDA animals in the caudate (D) and hippocampus (F, $\mathbf{f}$ ). HLA-DR-ir blood vessel scores were significantly higher in the caudate, septum, and hippocampus of 6-OHDA-treated animals compared with controls (G). Scores for HLA-DR-ir blood vessels negatively correlated with TH-ir nigral cell counts $(\mathbf{H}) . * P<0.05$. Scale bar: $(\mathbf{A}$ and $\mathbf{B})=10 \mathrm{~mm},(\mathbf{C}-\mathbf{F})=500 \mu \mathrm{m}$; insets $=100 \mu \mathrm{m}$.

Abbreviations: 6-OHDA, 6-hydroxydopamine; BV, blood vessel; HLA, human leukocyte antigen; TH, tyrosine hydroxylase; ir, immunoreactivity.

neurotransmitter, 6-OHDA is water-soluble and cannot cross the blood-brain barrier. Depending on the route of administration, the neurotoxin reaches central or peripheral catecholaminergic neurons. It actively enters these neurons through monoamine transporters and induces cell loss by increasing reactive oxygen species and blocking mitochondrial function. . $^{5,25,26}$

Although it is generally accepted that 6-OHDA does not cross the blood-brain barrier, previous studies have demonstrated mixed results on the effect of monoamine systems in the rodent central nervous system after the peripheral administration of 6-OHDA, specifically in young animals that do not have a fully developed blood-brain barrier. Systemic 6-OHDA dosing to mice and rats at birth and again at 48 hours $(2.5-10 \mathrm{mg} / \mathrm{kg}$ intraperitoneally) did not affect noradrenaline or dopamine concentrations in brain tissue as early as postnatal day 3 or as long as postnatal 60 days. ${ }^{27}$ A similar lack of effect in the hypothalamus, brainstem, and 

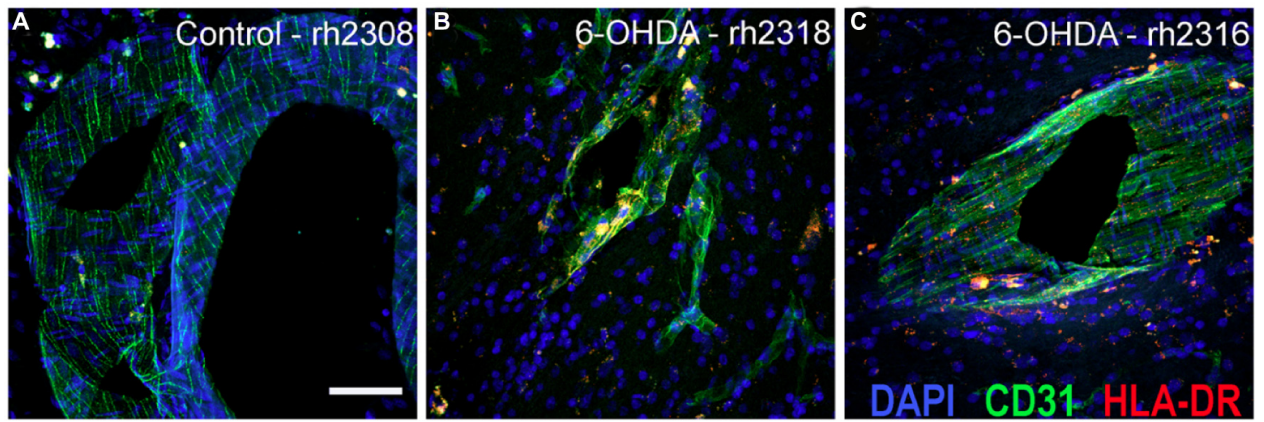

Figure 5 Immunofluorescent staining localizes HLA-DR-ir in CD3 I+ vasculature. Immunofluorescence labeling demonstrated increased HLA-DR-ir threaded in CD3 I-positive blood vessels of the ventral putamen in animals that received systemic 6-OHDA (B and C) compared with controls $(\mathbf{A})$. Scale bar $=25 \mu \mathrm{m}$.

Abbreviations: 6-OHDA, 6-hydroxydopamine; CD3I, platelet endothelial cell adhesion molecule; DAPI, 4',6-diamidino-2-phenylindole; HLA, human leukocyte antigen; ir, immunoreactivity.

cerebral hemispheres at 4 weeks postnatally was shown in rats that received 6 -OHDA ( $50 \mu \mathrm{g} / \mathrm{g} \times 7$ subcutaneously) within the first 10 hours after birth. ${ }^{28}$ Contrary to these reports, newborn mice and rats administered systemic 6-OHDA $(50 \mu \mathrm{g} / \mathrm{g} \times 4$ subcutaneously) and followed for 1,3 , and 6 months showed reduced labeled $\mathrm{H}^{3}$-noradrenaline $\left(30 \%-60 \%\right.$ of controls) in the brain. ${ }^{29}$ In another study, young kittens (age not defined) administered a single high dose of systemic 6-OHDA (20-30 mg/kg intraperitoneally) had reduced noradrenaline content in the hypothalamus 8 days post-toxin (no other central nervous system tissue was evaluated). ${ }^{6}$ In the same study, adult mice administered 6-OHDA ( $6.7 \mathrm{mg} / \mathrm{kg}$ intraperitoneally) did not have changes in dopamine or noradrenaline content in the brain 16 hours post toxin. These results suggest that age and toxin regimen may influence the effects of systemic 6-OHDA.

The subjects in this study received intravenous 6-OHDA, which induced peripheral catecholaminergic neuronal cell death, including loss of cardiac sympathetic innervation and dysautonomia, which were previously reported. ${ }^{17,18}$ These animals did not develop parkinsonian motor signs, and analyzed as a group, their TH-positive striatal fibers and nigral neurons were not significantly different to controls, which agrees with previous reports in adult rodents. ${ }^{27,28}$ Our rationale for the interindividual analysis was based on our observation in two subjects of atrophic morphology and decreased number of TH-ir neurons. In our experience, these characteristics are not in the range of what is expected in an unaffected dopaminergic nigral cell population, and looked similar to a neurotoxic effect. ${ }^{30}$ After exhaustive review of the medical history of 6-OHDA-treated animals, no notable procedures were completed on these monkeys prior to neurotoxin administration that would explain any differential effect on the nigrostriatal system or compromise of the blood-brain barrier. Rather, the individual variability resembles previous reports of subjects' responses to neurotoxins, in particular after systemic dosing. ${ }^{30,31}$

$\alpha$-Synuclein is a presynaptic protein of great interest in Parkinson's disease research, as its accumulation and aggregation have been associated with Parkinson's disease pathology, and mutations in the $\alpha$-synuclein gene have been found in cases of familial Parkinson's disease. ${ }^{32}$ $\alpha$-Synuclein-ir has been identified in the substantia nigra of baboons, ${ }^{14}$ squirrel monkeys, ${ }^{15}$ and rhesus monkeys, ${ }^{33}$ forming part of the dense network of terminal-like structures with limited expression in the nigral cell bodies. Interestingly, administration of the dopaminergic neurotoxin MPTP to baboons $^{14}$ and squirrel monkeys ${ }^{15}$ shifted the $\alpha$-synuclein location from fibers to the cell bodies. Similar pattern of $\alpha$-synuclein translocation into the cell body has been found in middle-aged (15-23 years) and aged rhesus monkeys (24-34 years) compared with young rhesus monkeys (2-12 years). ${ }^{33}$

In our study, the control adult rhesus monkeys had intense nigral $\alpha$-synuclein-ir in fibers and variable amounts in cell bodies, with the exception of one subject. Compared with the rest of the animals that were younger adults (5.2-9.1 years), this 17-year-old monkey showed low fiber but intense cell body expression, which agrees with reports of changes in intracellular distribution of $\alpha$-synuclein-ir with age. ${ }^{33}$ $\alpha$-Synuclein-ir in 6-OHDA-treated monkeys was low in nigral fibers, suggesting a link between neurotoxin exposure and location of $\alpha$-synuclein-ir, similar to reports with MPTP. ${ }^{14,15,33}$ Our finding of a positive correlation between the presence of $\alpha$-synuclein-ir in nigral cell bodies and the volume of TH-ir nigral cells suggests that the larger dopaminergic neurons maintain more $\alpha$-synuclein in the cell body in adult rhesus monkeys.

Although we did not find significant changes in the dopaminergic nigrostriatal system between groups, markers 
of inflammation were upregulated in cerebrovasculature 3 months post neurotoxin and negatively correlated with nigral TH-ir cell number, suggesting that 6-OHDA systemic dosing may indirectly affect the central nervous system. In rodents, 6-OHDA administration is associated with an upregulated glial response, ${ }^{34-36}$ while MPTP also elicits infiltration of peripheral macrophages or leukocytes. ${ }^{37}$ In rhesus monkeys, MPTP-induced reactive microglia have been reported to persist months ${ }^{38}$ to years ${ }^{39}$ after intoxication, yet in this study we did not find a change in ramified reactive microglia between groups. Although the link between neurotoxin-induced nigral cell loss and neuroinflammation is well documented, it is not clear if microglial markers are found in the cerebrovasculature after neurotoxin challenge, which could imply alteration of the blood-brain barrier.

Active neuroinflammation and alterations in the bloodbrain barrier are likely components of neurodegeneration in Parkinson's disease. ${ }^{16}$ Although the relationship between peripheral immune system and central neurodegeneration is not well understood, there is evidence that exposure to environmental factors including infections, which could trigger immune response and affect the blood-brain barrier, may contribute to Parkinson's disease etiology or progression. ${ }^{40,41}$ Disruption of the blood-brain barrier creates an opportunity for potential toxins to infiltrate the brain and contribute to Parkinson's disease progression. Previous studies in rodents have documented that 6-OHDA can affect blood-brain barrier integrity and allow transport of large molecules. Rats that received a unilateral injection of the neurotoxin into the striatum or the medial forebrain bundle followed 10 or 30 days later by intravenous fluorescein isothiocyanatelabeled albumin ${ }^{16}$ had albumin "hot spots" in areas of the striatum and mesencephalon ipsilateral to the 6-OHDA injection. In our study, 6-OHDA dosing may have affected the blood-brain barrier, allowing entry into the brain of small amounts of the neurotoxin, yet this is unlikely because of the high polarity of 6-OHDA. More likely, 6-OHDA toxicity may have increased oxygen or nitrogen species, that could have triggered the release of proinflammatory cytokines by the capillary endothelial cells of the blood-brain barrier, which have been described as a signaling interface between the brain and the blood. ${ }^{42}$ The release of these inflammatory mediators can destabilize the tight junctions between endothelial cells and compromise the integrity of the bloodbrain barrier. ${ }^{43}$ Ultimately, prolonged inflammation can lead to neurodegeneration, ${ }^{44}$ and may be responsible for the variable nigral cell counts found in the 6-OHDA animals in this study. Increased cytokines and chemokines can also recruit inflammatory cells and induce upregulation of adhesion molecules on the blood-brain barrier that control cell migration across this barrier. ${ }^{45,46}$ In this study, upregulation of HLA-DR in the blood vessels suggests that major histocompatibility complex II cells were recruited and remained tethered to the lining of the cerebral microvasculature due to limited entry into the brain. Future studies investigating the integrity of the blood-brain barrier and the presence of inflammatory mediators in the brain following systemic 6-OHDA are warranted.

In conclusion, our results demonstrate that systemic dosing of 6-OHDA in nonhuman primates does not produce motor deficits, but contrary to previous reports, it variably affects the dopaminergic nigrostriatal system and upregulates HLA-DR-ir in the cerebrovasculature.

\section{Acknowledgments}

This work was supported by the Wisconsin National Primate Research Center National Institutes of Health (WNPRC NIH) base grant P51RR000167, R21NS084158, University of Wisconsin-Madison National Institutes of Health Clinical and Translational Science Award (UW NIH-CTSA) pilot project award, and awards from the Graduate School of the University of Wisconsin, Madison. The authors would like to thank Dr Vita Bondarenko for excellent technical assistance with immunohistochemical procedures, and Dr Marie-Francoise Chesselet and Chunni Zhu for helpful discussions.

\section{Disclosure}

The authors report no conflict of interest in this work.

\section{References}

1. Forno LS, DeLanney LE, Irwin I, Di Monte D, Langston JW. Astrocytes and Parkinson's disease. Prog Brain Res. 1992;94:429-436.

2. McGeer PL, Itagaki S, Boyes BE, McGeer EG. Reactive microglia are positive for HLA-DR in the substantia nigra of Parkinson's and Alzheimer's disease brains. Neurology. 1988;38:1285-1291.

3. Mirza B, Hadberg H, Thomsen P, Moos T. The absence of reactive astrocytosis is indicative of a unique inflammatory process in Parkinson's disease. Neuroscience. 2000;95:425-432.

4. Vila M, Jackson-Lewis V, Guegan C, et al. The role of glial cells in Parkinson's disease. Curr Opin Neurol. 2001;14:483-489.

5. Kostrzewa RM, Jacobowitz DM. Pharmacological actions of 6-hydroxydopamine. Pharmacol Rev. 1974;26:199-288.

6. Laverty R, Sharman DF, Vogt M.Action of 2,4,5-trihydroxyphenylethylamine on the storage and release of noradrenaline. Br J Pharmacol Chemother. 1965;24:549-560.

7. Eslamboli A, Baker HF, Ridley RM, Annett LE. Sensorimotor deficits in a unilateral intrastriatal 6-OHDA partial lesion model of Parkinson's disease in marmoset monkeys. Exp Neurol. 2003;183:418-429.

8. Ichitani Y, Okamura H, Matsumoto Y, Nagatsu I, Ibata Y. Degeneration of the nigral dopamine neurons after 6-hydroxydopamine injection into the rat striatum. Brain Res. 1991;549:350-353. 
9. Marinova-Mutafchieva L, Sadeghian M, Broom L, Davis JB, Medhurst AD, Dexter DT. Relationship between microglial activation and dopaminergic neuronal loss in the substantia nigra: a time course study in a 6-hydroxydopamine model of Parkinson's disease. J Neurochem. 2009;110:966-975.

10. Rodriguez-Pallares J, Parga JA, Munoz A, Rey P, Guerra MJ, Labandeira-Garcia JL. Mechanism of 6-hydroxydopamine neurotoxicity: the role of NADPH oxidase and microglial activation in 6-hydroxydopamine-induced degeneration of dopaminergic neurons. J Neurochem. 2007;103:145-156.

11. Roeling TA, Docter GJ, Voorn P, Melchers BP, Wolters EC, Groenewegen HJ. Effects of unilateral 6-hydroxydopamine lesions on neuropeptide immunoreactivity in the basal ganglia of the common marmoset, Callithrix jacchus, a quantitative immunohistochemical analysis. J Chem Neuroanat. 1995;9:155-164.

12. Sauer H, Oertel WH. Progressive degeneration of nigrostriatal dopamine neurons following intrastriatal terminal lesions with 6-hydroxydopamine: a combined retrograde tracing and immunocytochemical study in the rat. Neuroscience. 1994;59:401-415.

13. Joers V, Emborg ME. Modeling and imaging cardiac sympathetic neurodegeneration in Parkinson's disease. Am J Nucl Med Mol Imaging. 2014;4:125-159.

14. Kowall NW, Hantraye P, Brouillet E, Beal MF, McKee AC, Ferrante RJ. MPTP induces alpha-synuclein aggregation in the substantia nigra of baboons. Neuroreport. 2000;11:211-213.

15. Purisai MG, McCormack AL, Langston WJ, Johnston LC, Di Monte DA. Alpha-synuclein expression in the substantia nigra of MPTP-lesioned non-human primates. Neurobiol Dis. 2005;20:898-906.

16. Carvey PM, Hendey B, Monahan AJ. The blood-brain barrier in neurodegenerative disease: a rhetorical perspective. J Neurochem. 2009;111:291-314.

17. Joers V, Dilley K, Rahman S, et al. Cardiac Sympathetic Denervation in 6-OHDA-Treated Nonhuman Primates. PLoS One. 2014;9:e104850.

18. Joers V, Seneczko K, Goecks NC, et al. Nonuniform cardiac denervation observed by $11 \mathrm{C}$-meta-hydroxyephedrine PET in 6-OHDAtreated monkeys. PLoS One. 2012;7:e35371.

19. Swanson C, Emborg M. Expression of peroxisome proliferatoractivated receptor-gamma in the substantia nigra of hemiparkinsonian nonhuman primates. Neurol Res. 2014;36:634-646.

20. Emborg ME, Ma SY, Mufson EJ, et al. Age-related declines in nigral neuronal function correlate with motor impairments in rhesus monkeys. J Comp Neurol. 1998;401:253-265.

21. Emborg ME, Moirano J, Schafernak KT, et al. Basal ganglia lesions after MPTP administration in rhesus monkeys. Neurobiol Dis. 2006;23:281-289.

22. Emborg ME, Liu Y, Xi J, et al. Induced pluripotent stem cell-derived neural cells survive and mature in the nonhuman primate brain. Cell Rep. 2013;3:646-650.

23. Ohshima-Hosoyama S, Simmons HA, Goecks N, et al. A monoclonal antibody-GDNF fusion protein is not neuroprotective and is associated with proliferative pancreatic lesions in parkinsonian monkeys. PLoS One. 2012;7:e39036.

24. Emborg ME, Zhang Z, Joers V, et al. Intracerebral transplantation of differentiated human embryonic stem cells to hemiparkinsonian monkeys. Cell Transplant. 2013;22:831-838.

25. Blandini F, Armentero MT, Martignoni E. The 6-hydroxydopamine model: news from the past. Parkinsonism Relat Disord. 2008;14 Suppl 2: S124-S129.

26. Schober A. Classic toxin-induced animal models of Parkinson's disease: 6-OHDA and MPTP. Cell Tissue Res. 2004;318:215-224.

27. Lytle LD, Shoemaker WJ, Cottman K, Wurtman RJ. Long-term effects of postnatal 6-hydroxydopamine treatment on tissue catecholamine levels. J Pharmacol Exp Ther. 1972;183:56-64.
28. Jaim-Etcheverry G, Zieher LM. Permanent depletion of peripheral norepinephrine in rats treated at birth with 6-hydroxydopamine. Eur J Pharmacol. 1971;13:272-276.

29. Angeletti PU. Chemical sympathectomy in newborn animals. Neuropharmacology. 1971;10:55-59.

30. Emborg ME. Nonhuman primate models of Parkinson's disease. ILAR J. 2007;48:339-355.

31. Emborg ME. Evaluation of animal models of Parkinson's disease for neuroprotective strategies. J Neurosci Methods. 2004;139:121-143.

32. Bendor JT, Logan TP, Edwards RH. The function of alpha-synuclein. Neuron. 2013;79:1044-1066.

33. Chu Y, Kordower JH. Age-associated increases of alpha-synuclein in monkeys and humans are associated with nigrostriatal dopamine depletion: is this the target for Parkinson's disease? Neurobiol Dis. 2007;25:134-149.

34. Cicchetti F, Brownell AL, Williams K, Chen YI, Livni E, Isacson O. Neuroinflammation of the nigrostriatal pathway during progressive 6-OHDA dopamine degeneration in rats monitored by immunohistochemistry and PET imaging. Eur J Neurosci. 2002;15:991-998.

35. Depino AM, Earl C, Kaczmarczyk E, et al. Microglial activation with atypical proinflammatory cytokine expression in a rat model of Parkinson's disease. Eur J Neurosci. 2003;18:2731-2742.

36. Stromberg I, Bjorklund H, Dahl D, Jonsson G, Sundstrom E, Olson L. Astrocyte responses to dopaminergic denervations by 6-hydroxydopamine and 1-methyl-4-phenyl-1,2,3,6-tetrahydropyridine as evidenced by glial fibrillary acidic protein immunohistochemistry. Brain Res Bull. 1986;17:225-236.

37. Kurkowska-Jastrzebska I, Wronska A, Kohutnicka M, Czlonkowski A, Czlonkowska A. The inflammatory reaction following 1-methyl-4phenyl-1,2,3, 6-tetrahydropyridine intoxication in mouse. Exp Neurol. 1999; 156:50-61.

38. Swanson CR, Joers V, Bondarenko V, et al. The PPAR-gamma agonist pioglitazone modulates inflammation and induces neuroprotection in parkinsonian monkeys. J Neuroinflammation. 2011;8:91.

39. McGeer PL, Schwab C, Parent A, Doudet D. Presence of reactive microglia in monkey substantia nigra years after 1-methyl-4-phenyl1,2,3,6-tetrahydropyridine administration. Ann Neurol. 2003;54: 599-604.

40. Engler H, Doenlen R, Riether C, et al. Time-dependent alterations of peripheral immune parameters after nigrostriatal dopamine depletion in a rat model of Parkinson's disease. Brain Behav Immun. 2009;23:518-526.

41. Villaran RF, Espinosa-Oliva AM, Sarmiento M, et al. Ulcerative colitis exacerbates lipopolysaccharide-induced damage to the nigral dopaminergic system: potential risk factor in Parkinson's disease. J Neurochem. 2010;114:1687-1700.

42. Verma S, Nakaoke R, Dohgu S, Banks WA. Release of cytokines by brain endothelial cells: a polarized response to lipopolysaccharide. Brain Behav Immun. 2006;20:449-455.

43. Burns TC, Verfaillie CM, Low WC. Stem cells for ischemic brain injury: a critical review. J Comp Neurol. 2009;515:125-144.

44. Gendelman HE. Neural immunity: friend or foe? J Neurovirol. 2002;8: 474-479.

45. Owens T, Bechmann I, Engelhardt B. Perivascular spaces and the two steps to neuroinflammation. J Neuropathol Exp Neurol. 2008;67:1113-1121.

46. Barcia C, Emborg ME, Hirsch EC, Herrero MT. Blood vessels and parkinsonism. Front Biosci. 2004;9:277-282. 
Journal of Inflammation Research

\section{Publish your work in this journal}

The Journal of Inflammation Research is an international, peer-reviewed open-access journal that welcomes laboratory and clinical findings on the molecular basis, cell biology and pharmacology of inflammation including original research, reviews, symposium reports, hypothesis formation and commentaries on: acute/chronic inflammation; mediators of inflamma-
Dovepress

tion; cellular processes; molecular mechanisms; pharmacology and novel anti-inflammatory drugs; clinical conditions involving inflammation. The manuscript management system is completely online and includes a very quick and fair peer-review system. Visit http://www.dovepress.com/ testimonials.php to read real quotes from published authors.

Submit your manuscript here: http://www.dovepress.com/journal-of-inflammation-research-journal 